\title{
Influence of drop velocity and droplet spacing on the equilibrium saturation level in binder jetting
}

\author{
Trenton Colton*, Joseph Liechty*, Alden McLean*, Nathan Crane* \\ *Department of Mechanical Engineering, Brigham Young University, Provo, UT
}

\begin{abstract}
$\underline{\text { Abstract }}$
Understanding the equilibrium saturation level is crucial to Binder Jetting (BJ). Saturation level influences dimensional accuracy, print time, green strength, and final material properties. Improved understanding of the saturation level can reduce development time for new materials and improve existing processes in BJ. Attempts have been made to predict saturation levels of parts with simple calculations from droplet primitives and capillary pressure. There is, however, limited experimental validation for these methods and they do not include the impact of drop velocity and droplet spacing. This study incorporates the influences of drop velocity and droplet spacing on the saturation level of the part. Drop primitives of varying droplet velocity and droplet spacing were compared. Results show that velocity impacts the feasible parameter space.
\end{abstract}

\section{Keywords:}

Additive Manufacturing, Binder Jetting.

\section{Introduction}

Binder Jetting (BJ) is a low-cost Additive Manufacturing (AM) process that uses a particle bed and inkjet technology. The powder is dispensed in thin layers and using inkjet nozzles to selectively bind powder in a chosen cross section, this is repeated layer by layer [1]. Similar to other powder bed AM methods such as Selective Laser Sintering (SLS), Electron Beam Melting (EBM), etc., BJ uses unbound powder from lower levels as a support structure for upper layers. BJ can utilize various materials such as ceramics, metals, and polymers [2]. For BJ to accelerate its implementation in industry; steps need to be taken to improve dimensional accuracy, improve mechanical properties, and decrease cost. These current limitations are influenced by the binderpowder interaction. Better understanding of the fundamental parameters of binder-powder interaction will decrease these limitations. Proper parameters for a new environment or new material can be identified quickly without extensive experimentation.

The key to BJ is the binder-powder interaction. In BJ, 40-60 $\mu$ m droplets impact powder with velocities ranging 1-10 m/s. The forces in the interaction are complex. Both capillary and inertial forces are significant and powder rearrangement is typical. In this work the influence of velocity and droplet spacing in the binder-powder interaction are investigated. Experiments have shown the interaction can impact dimensional accuracy, porosity, and strength [3].

Binder saturation level measures the amount of void space in the powder filled by binder. Equilibrium saturation is the amount of binder that is stable in a powder bed. It is generally accepted that the binder saturation level in BJ should be below the equilibrium saturation level. Equilibrium saturation is a function of the binder, powder (size, shape), and pore size in the powder bed [4]. While saturation is typically taken as a single parameter, there is actually a range of equilibrium values due to hysteresis between the imbibition and drainage curves [5]. Printing saturation level is the ratio of the amount of binder per volume of powder to the porosity per volume of powder. 
Printhead speed has been shown to alter the saturation level and decrease dimensional accuracy in printed parts [6]. Printhead speed alters the droplet velocity and direction. Higher printhead speed increases splashing, rebounding, and cratering. The saturation level estimated from simple calculations based on binder drop primitives and capillary pressure have little experimental validation and do not include the impact of velocity [4, 7]. An alternate approach to understanding the interaction is in situ observation. Studies conducted using in situ high-speed imaging and x-ray imaging have observed deformation of the powder bed and primitive formation $[8,9]$.

In this study, we characterize the influence of droplet velocity and droplet spacing on the equilibrium saturation level. Experiments performed utilizing sessile drop primitives, and printed lines characterize the dependence of equilibrium saturation levels on droplet velocity and droplet spacing.

\section{Fluid Flow in Powder}

Much of the current understanding of the binder-powder interaction physics is based on the flow of fluid in soil. Bredt discussed the challenge presented by the scale between soil hydrology and BJ [5]. The structures in soil hydrology can be quite large and models are not bounded by time which exaggerate the predicted size of printed features. Bredt's model assumed no kinetic impact, no hysteresis in the advancing or receding of droplet contact angles, and no evaporation effect [5]. Binder will migrate through voids in powder until the pressure across the binder-air interface is in equilibrium. The curvature of the liquid interfaces in the pores creates a pressure difference across the interface. The pressure difference varies with saturation level.

In BJ, one point on the surface is initially fully saturated. Unsaturated regions absorb binder as capillary pressure drives flow from saturated regions until equilibrium is reached. Due to powder packing variation and the hysteresis in the imbibition and drainage curves the saturation level will be slightly different throughout the wetted region. Observations of the droplet impact such as cratering and particle movement contribute to undesirable part defects [8, 9]. This deformation of powder beds and impact of kinetic energy can be seen in the overall part quality and dimensional tolerance. Saturation decreased and inaccuracies increased with printhead speed [6].

The prediction of saturation level in $\mathrm{BJ}$ is valuable in decreasing time to identify appropriate printing parameters. Miyanaji [7] proposed the first physics-based model to predict the equilibrium saturation level based on the measured capillary pressure. The model assumes a single equivalent pore size which amounts to modeling the powder bed as a series of cylindrical pores of the same size. Problems with this model occurred when successive layers were printed the lower layers became more saturated. It was speculated to be caused by drainage from the upper layers [7]. Unfortunately, the limited experimental data set did not show consistent correlation between model predictions of equilibrium saturation and part-level measurements. Miyanaji attributed the difference to errors in powder property measurement. However, this model does not account for parameters such as the impact of droplet velocity in BJ printing or the range of pore sizes in a powder bed or the effects of binder evaporation between printed layers. 
Bai (2019) characterized the binder-powder interaction by studying the primitives formed by millimeter-scale sessile drops. The study calculated the dynamic contact angle from measured imbibition times of sessile drops and attempted to correlate these measurements to saturation level [4]. Bai noted that droplet velocity was a variable to account for in future testing. The sessile drop is limited in predicting the binder-powder interaction in BJ because it did not account for effects of kinetic energy. Predicting the interaction is valuable and can improve with a greater understanding of kinetic energy and droplet spacing in BJ.

\section{$\underline{\text { Methods }}$}

The characterization of the binder-powder interaction was studied through two experimental geometries. Initial binder mass and the mass of the resultant primitives were measured to calculate equilibrium saturation levels based on the binder mass $\left(m_{b}\right)$ and the primitive mass $\left(m_{p}\right)$, using:

$$
S=\frac{m_{p}}{{\frac{m_{b}}{p_{p b}}}^{*}(1-P)}
$$

where $p_{b}$ is binder density and $p_{p b}$ is powder bed density, and $P_{f}$ is the packing fraction. The experimental geometries isolated the effects of velocity and droplet spacing. The BJ experimental apparatus was designed and built to control droplet velocity and droplet spacing independently for repeatable experiments.

The experiments were conducted using ExOne stainless steel 420 powder with an average particle size of $30 \mu \mathrm{m}$. Powder beds were prepared using aluminum containers filled with powder and tapped. The surface was then leveled using a straight edge. The tapped density was approximately $4.66 \mathrm{~g} / \mathrm{cm}^{3}$. This equates to a packing fraction of 0.58 . Density was calculated by measuring the mass required to fill a container with a known volume. Powder beds were prepared in this manner in all experiments.

The two experimental procedures are outlined as (1) primitive formation from droplets released from varying heights to vary velocity. (2) Line printing with varying velocities and droplet spacing.

The first experiment procedure, primitive droplet formation was measured by dropping millimeter-scaled droplets (2-14.5 microliters) into a powder bed from controlled heights to control the droplet velocity. Binder was dispensed from a $5 \mathrm{ml}$ syringe through a needle. The droplet volume was measured by placing the powder bed on the scale (Ohaus AX224) to measure the mass of each droplet as it was deposited as illustrated in Figure 1. To average effects of any powder bed density variation within the bed, droplet primitive placement was varied for each droplet release height. Drops were released at heights of 0,10 , and $20 \mathrm{~cm}$ directly over the scale allowing each drop mass to be measured accurately [10]. Larger release heights caused splashing. ExOne solvent binder was used and volume was calculated from mass measurements. The ExOne solvent binder had a measured density of $0.965 \mathrm{~g} / \mathrm{cm}^{3}$. The powder beds were heated in an oven at 180 degrees Celsius for 2 hours immediately after. The primitives' masses were measured to calculate the 
equilibrium saturation level from Equation 1. This ratio normalized the values and allows for a comparison with the range of values.

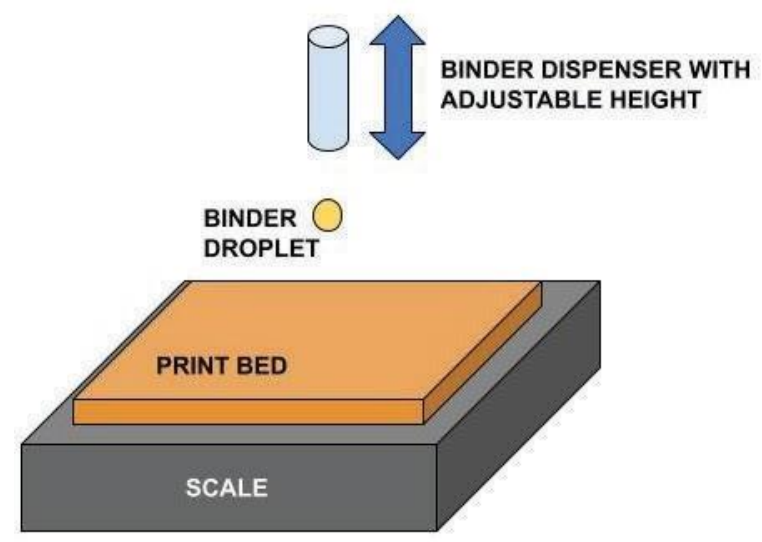

Figure 1: Apparatus design for droplet primitive experiment. The droplet was dropped from controlled heights above the scale. By controlling the height, the velocity of the droplet changed. Heights of 0,10 , and $20 \mathrm{~cm}$ were included in the test. Heights above $20 \mathrm{~cm}$ splashed.

The second experimental procedure, the custom apparatus for line printing is shown in Figure 2. The apparatus consisted of MicroFab MJ-AB-01 single nozzle (40 and $60 \mu \mathrm{m}$ orifice) print setup in unison with three motorized linear stages. The MicroFab single nozzle print setup utilized a MicroFab pneumatics control system, binder reservoir, tubing, and a piezoelectric nozzle. The binder reservoir is pressurized by the pneumatics console and maintained at the same height relative to the printhead to prevent pressure difference during movement of the printhead.

The piezoelectric nozzle ejects droplets when an electric waveform signal is received from the drive box (Jet Drive III). Figure 3 shows the integration of the various systems. The droplet mass and initial velocity are dependent on the voltage and timing of the waveform. The general waveform used is known as a bipolar waveform [11]. Droplet velocity was changed by altering the voltage of the waveform. Droplet images were produced from IDS UI-3370CP-M-GL camera at 2.5 magnification and a strobing LED. Distance measured between droplet images created by successive strobes flashes were used to calculated droplet velocity.

A custom powder bed container was made to attach to two linear motor stages. ExOne solvent binder was used for the experiment. The inkjet nozzle was triggered in unison with the movement of the linear motor creating printed lines with the powder. To determine the amount of binder used, the system ran twenty lines, twenty $\mathrm{mm}$ in length with a small container underneath to collect binder. The mass of dispensed binder was measured. After printing, the powder bed was heated at 180 degrees Celsius for thirty minutes in an oven. Each individual lines' mass was measured to calculate the effective saturation level as a function of droplet velocity and droplet spacing using Equation 1. 


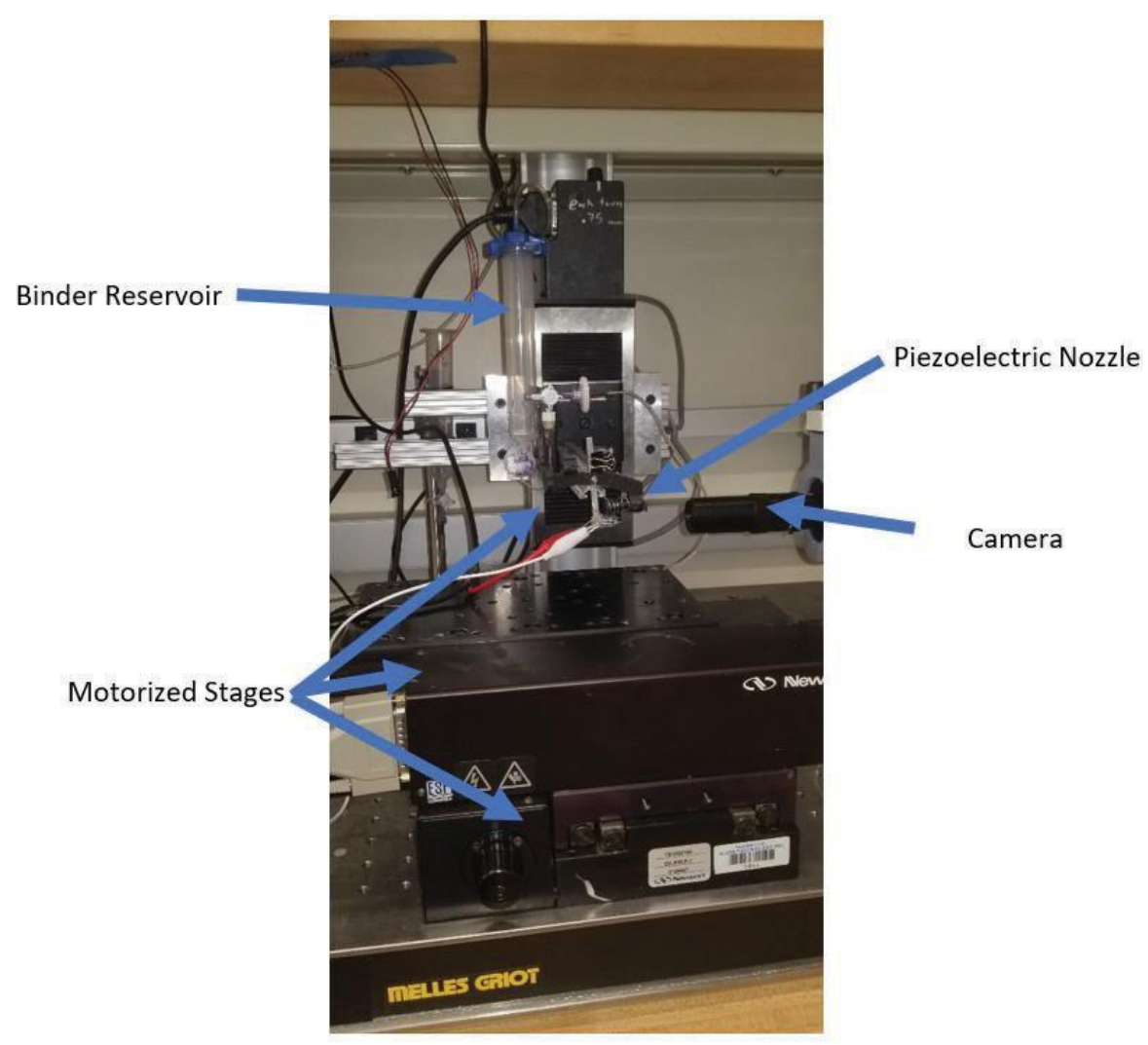

Figure 2: Image of the experimental apparatus for line printing. Shown are stages for bed and printhead movement.

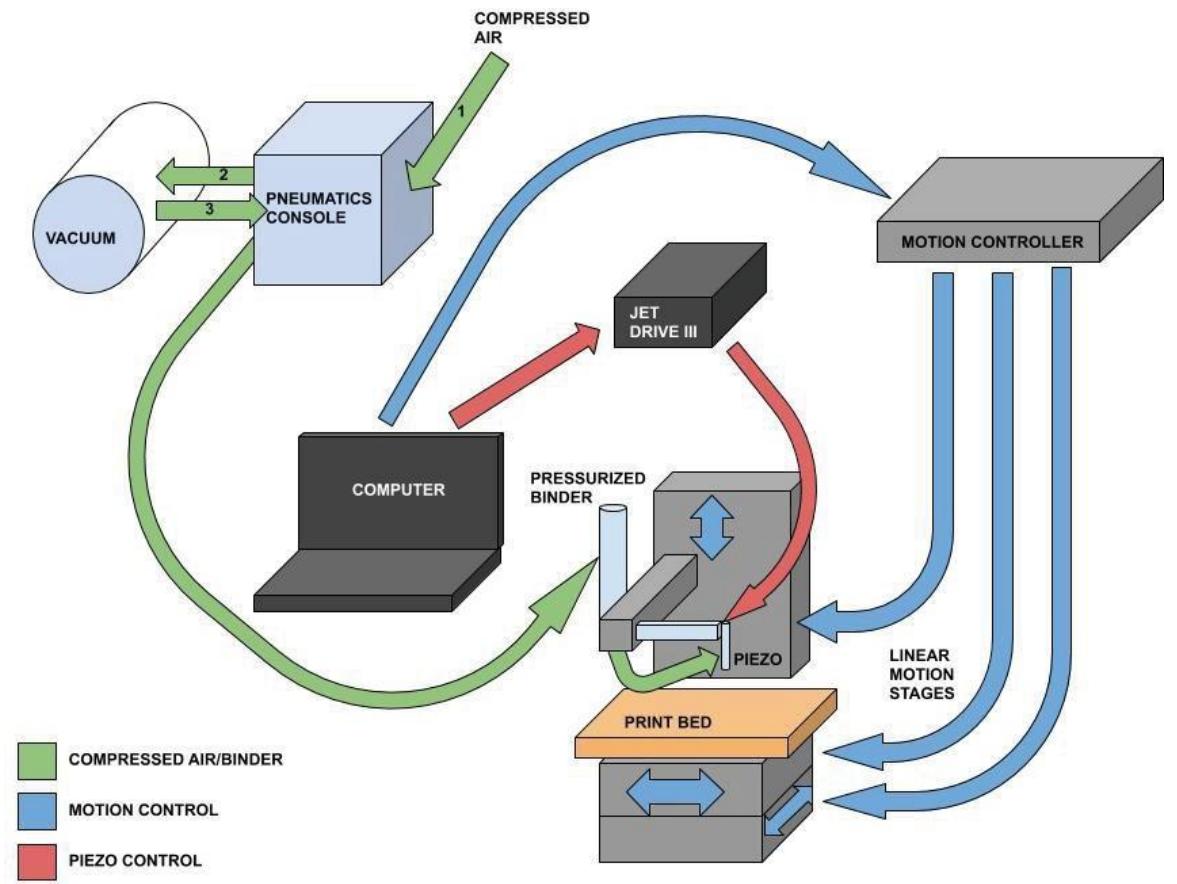

Figure 3: A diagram of the experimental apparatus and connectivity between units. A LabVIEW VI drove the Newport linear stages in unison with triggering the JetDrive III for control of the printhead. 


\section{$\underline{\text { Results and Discussion }}$}

The equilibrium saturation of drops from the first experimental procedure (Figure 1) is summarized in Figure 4. millimeter-scaled droplets (2-14.5 microliters) with masses ranging 0.002-0.014 grams were drop heights were 0,10 , and $20 \mathrm{~cm}$. The equilibrium saturation level ranged between $65 \%$ to $95 \%$ with significant variation in each measurement. The saturation level is relatively insensitive to drop height suggesting that increasing velocity did not change effective saturation levels for these millimeter-scale droplets. The variation of saturation levels at each height may be attributed to variation of powder bed density within the powder bed or changes in environmental conditions. However, there may be significant differences in the physics of the interaction when comparing single mm-scale droplets to lines of inkjet-printed droplets where each droplet is <100 microns in diameter.

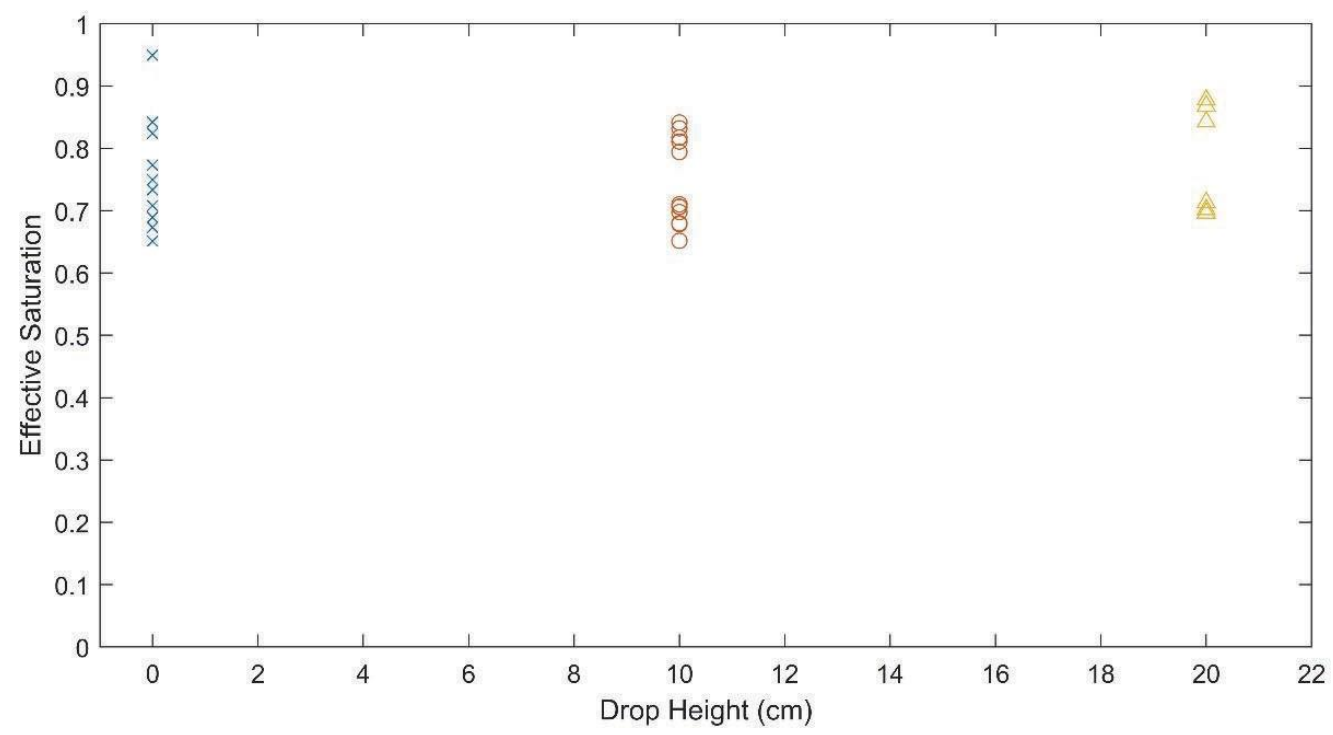

Figure 4: Saturation level of primitives from drops made using a syringe shown. No correlation is shown between low velocity and relatively large droplets.

Results from inkjet printing of lines are shown in Figure 5. The trend of higher saturation level at lower velocities can clearly be seen. There appears to be a limit at lower velocities approaching $80 \%$ saturation and upper velocities approaching 50\% saturation-well beyond the standard deviation in the measurements. The range of tested droplet velocity covers the typical seen in BJ systems. Satellite droplets, rebounding, and splashing could be affecting these saturation measurements, but no clear evidence of these defects was observed inspecting the powder bed after printing. However, magnified imaging of the powder bed or high-speed imaging during printing might provide additional insight. 


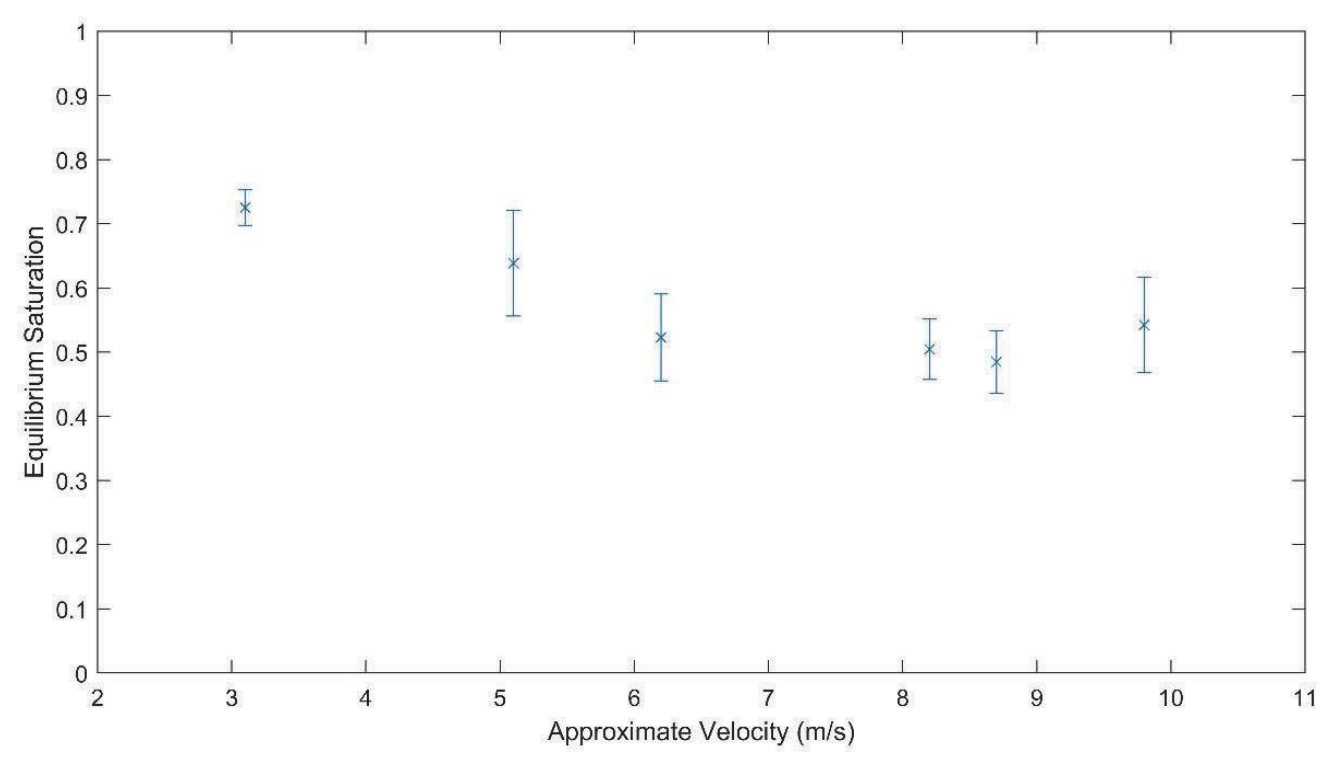

Figure 5: Results from lines printed with a 60-micron orifice print head at $2000 \mathrm{HZ}$ in 420 stainless steel powder with one standard deviation of the average shown in error bars. Trends to higher equilibrium saturation at lower velocities is seen. Droplet spacing was uniform through the experiment. Droplet shape, velocity, and size varied with the change in waveform. Error bars are small but show one standard deviation.

The decrease in effective saturation levels with increasing velocity shows that the kinetic energy of the droplet impacts the absorption characteristics of the droplet — at least in the case of a single line on bare powder. Millimeter-scale droplets falling $20 \mathrm{~cm}$ will have a velocity of approximately $2 \mathrm{~m} / \mathrm{s}$ if air resistance is negligible. The lower range of saturations in these mmscale droplets is comparable to the mean value of the lines printed with $3 \mathrm{~m} / \mathrm{s}$ droplets. However, the typical printing velocity is $>5 \mathrm{~m} / \mathrm{s}$ where the kinetic energy is observed to reduce the effective saturation levels of the binder in the powder.

The impact of droplet spacing on effective saturation levels is shown in Figure 6. Four separate data sets at different droplet velocity were recorded. A $40 \mu \mathrm{m}$ orifice was used and droplet spacings ranging between 5-12.5 $\mu \mathrm{m}$, droplet sizes ranging between 33-75 $\mu \mathrm{m}$ diameter. The trend of decreasing equilibrium saturation with increasing velocity generally holds with different droplet spacings though was broken with overlap at velocities 5.4 and $9.1 \mathrm{~m} / \mathrm{s}$. An increasing droplet spacing clearly decreased equilibrium saturation at all velocities.

The impact of increased velocity is decreased with larger droplet overlap as seen in Figure 6. Images of printed lines shown in Figure 7 demonstrated that the line width increased but the edge roughness stayed similar. Uneven edges may be caused by the tendency for binder to fill the smallest pores first due to the larger capillary pressure in small pores. Possible errors such as satellite drops, rebounding, and splashing could also be playing roles in this situation. However, there is good agreement between the two orifice sizes at same velocities. 


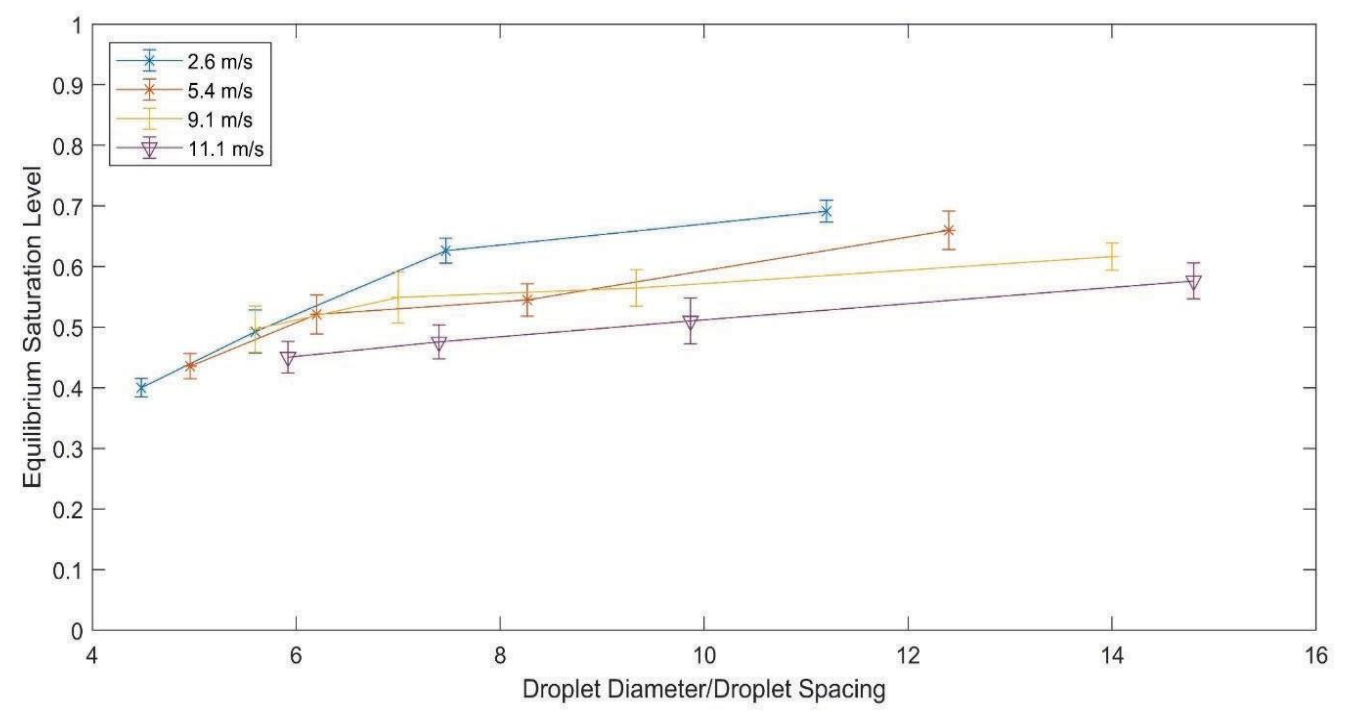

Figure 6: Results from differing droplet diameter/droplet spacing using a 40 micron orifice at $1000 \mathrm{HZ}$. One standard deviation of the average shown in error bars. The droplets were spaced between 5 and 12.5 microns apart. The ratio was used to normalize the effect of the changing volume from the different waveforms at each velocity.

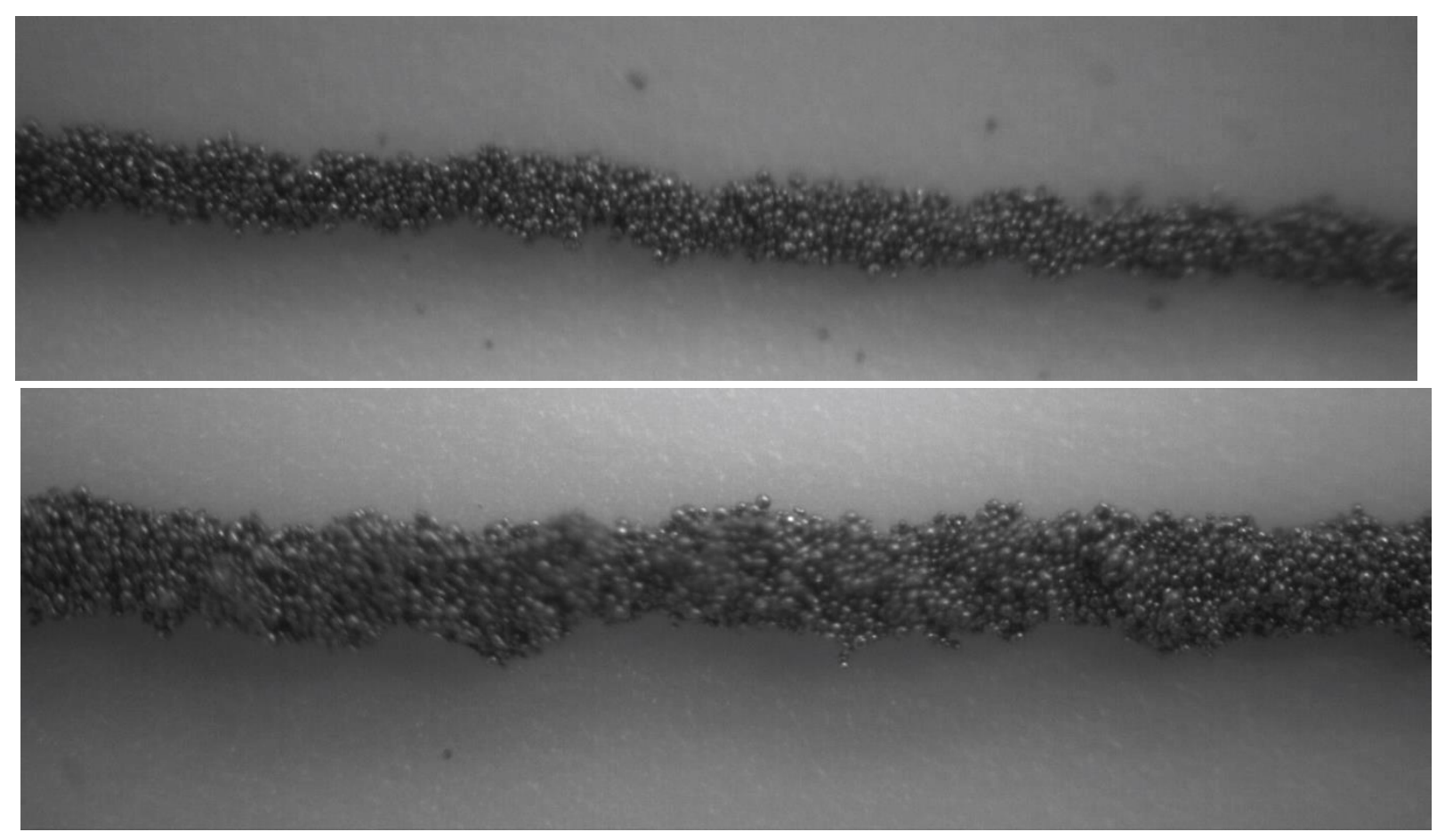

Figure 7: Bottom line is $4 \mathrm{~m} / \mathrm{s}$ droplets at $1000 \mathrm{HZ}$, droplet spacing of 12.5 microns. Top line shown is $4 \mathrm{~m} / \mathrm{s}$ at $1000 \mathrm{HZ}$, droplet spacing of 5 microns. 


\section{Conclusion}

The binder-powder interaction is complex. Increased understanding of the interaction will lead to improved processes and faster development of new materials. This study examined impact of velocity on the equilibrium saturation level. The few models currently predicting the saturation level disregard the impact of velocity and assume quasi-static conditions. This work shows that droplet velocity does have a significant impact on the equilibrium saturation level. In the test material system, increasing droplet velocity from $3 \mathrm{~m} / \mathrm{s}$ to $9 \mathrm{~m} / \mathrm{s}$ reduces saturation from $73 \%$ to $48 \%$. As improved powder printing models are developed the impact of velocity should be included and further researched.

Decreasing droplet spacing increases saturation towards the values characteristic of low velocity impacts. Similar effects may also be seen from printing adjacent lines or subsequent layers. The variation of saturation level with velocity and droplet spacing should be investigated further to assist in developing a more complete model of the binder-powder interaction. Observation of satellite drops, splashing, rebounding, and a changing waveform need to be investigated further to understand the mechanism for the changes. Experimental observations of other geometries such as multilayer and multiline parts could provide greater insight to characterize the interaction.

\section{Acknowledgment}

This work was funded in part by the National Science Foundation through award CMMI 1912028.

\section{References}

[1] L. E. Murr, "Frontiers of 3D Printing/Additive Manufacturing: from Human Organs to Aircraft Fabricationt," Journal of Materials Science \& Technology, vol. 32, no. 10, pp. 987-995, 2016/10/01/ 2016, doi: https://doi.org/10.1016/j.jmst.2016.08.011.

[2] Y. Bai, G. Wagner, and C. B. Williams, "Effect of Particle Size Distribution on Powder Packing and Sintering in Binder Jetting Additive Manufacturing of Metals," (in English), J. Manuf. Sci. Eng.Trans. ASME, Article vol. 139, no. 8, p. 6, Aug 2017, Art no. 081019, doi: 10.1115/1.4036640.

[3] Y. Bai, C. Wall, H. Pham, A. Esker, and C. B. Williams, "Characterizing Binder-Powder Interaction in Binder Jetting Additive Manufacturing Via Sessile Drop Goniometry," J. Manuf. Sci. Eng.-Trans. ASME, vol. 141, no. 1, Jan 2019, Art no. 011005, doi: 10.1115/1.4041624.

[4] J. Bredt, "Binder Stability and Powder/Binder Interaction in Three Dimensional Printing," MIT Thesis, 1995.

[5] H. Miyanaji, N. Momenzadeh, and L. Yang, "Effect of printing speed on quality of printed parts in Binder Jetting Process," Additive Manufacturing, vol. 20, pp. 1-10, Mar 2018, doi: 10.1016/j.addma.2017.12.008.

[6] H. Miyanaji, S. Zhang, and L. Yang, "A new physics-based model for equilibrium saturation determination in binder jetting additive manufacturing process," International Journal of Machine Tools and Manufacture, vol. 124, pp. 1-11, 2018, doi:

10.1016/j.ijmachtools.2017.09.001. 
[7] T. Fan, "Droplet-powder impact interaction in three dimentional printing," MIT Thesis, Thesis 1996.

[8] N. D. Parab et al., "Real time observation of binder jetting printing process using high-speed Xray imaging," Scientific Reports, vol. 9, no. 1, p. 2499, 2019/02/21 2019, doi: 10.1038/s41598019-38862-7.

[9] I. Gibson, D.W. Rosen, B. Stucker, Additive Manufacturing Technologies: 3D Printing, Rapid Prototyping, and Direct Digital manufacturing, 2nd., Springer, New York, (2015), pp. 1-498.

[10] T. Gao et al., "Granule formation and structure from single drop impact on heterogeneous powder beds," International Journal of Pharmaceutics, Article vol. 552, no. 1-2, pp. 56-66, 2018, doi: 10.1016/j.ijpharm.2018.09.036.

[11] "Microfab Technologies I. Ink-jet Microdispensor. A basic ink-jet setup.." 